\title{
Estimating Queue at Traffic Signals
}

\author{
Johannes J. Bezuidenhout, Prakash Ranjitkar* and Roger Dunn
}

\author{
Department of Civil and Environmental Engineering, University of Auckland, New Zealand
}

\begin{abstract}
This paper proposes a new queue prediction model based on the data that can be collected from a single loop detector positioned at the stop line of signalised intersections. A number of different model forms were explored using an enhanced NGSIM dataset. These data were filtered to represent the data that can be typically collected from a stop line detector loop. The best six models resulted in an accuracy ranging from $83 \%$ to $95 \%$ to correctly predict the state of vehicle's discharge close to the stop line that is whether it is a queued or platooned vehicle. When combined with a logical filter to group sequential vehicles, it enables a traffic controller to estimate the most likely queue length. The proposed model will form part of a new offset optimizer algorithm currently under development.
\end{abstract}

Keywords: Logistic regression, queue estimation, SCATS, single loop detectors.

\section{INTRODUCTION}

Optimising traffic signals often rely on inductive detector loops that can only provide limited data to signal controllers, from which it continually adjust green splits, cycle time, and offsets. However, effective offset times need to consider the time to discharge a downstream queue, and therefore an estimate of the next cycle's queue length becomes an important variable to optimise the system. Inductive loop detectors can only provide limited, point observed data, to a traffic control system, typically these being occupancy, volumes, headways and follow-up gaps between the vehicles as they pass over the loop.

Occupancy is the time a vehicle occupies the loop, measured from the time the front of the vehicle enters the detection zone to the time when the rear of the vehicle leaves the detection zone. Headway is the time to travel from the front centre of the vehicle (at the speed of the vehicle) to the front centre of the preceding vehicle. Follow-up gap is the time between the back of the leading vehicle and the front of the following vehicle measured in time at a single point of observation.

However, other traffic flow values such as vehicle speed can be derived using relationships between space-time and speed [1]. However, it is also necessary for the controller to know the effective length of a vehicle to enable an accurate prediction of speed. A heterogeneous traffic stream with different vehicle lengths reduces the accuracy.

A single loop detector at the stop line in isolation of other loops, cannot measure a queue on an intersection approach. This paper proposes a new method that relies only on a single inductive detector placed at the stop line to estimate the queue length based on only those traffic flow properties that it can detect when it discharges. The discharging queue

*Address correspondence to this author at the Department of Civil and Environmental Engineering, University of Auckland, Auckland, New Zealand; Tel: 619 9233515; Fax: 649 3737462;

E-mail: p.ranjitkar@auckland.ac.nz then serves as a proxy for estimating the next cycle's queue length, assuming that the flow conditions between the preceding and successive cycle do not change significantly.

The Sydney Coordinated Adaptive Traffic System (SCATS), which is widely deployed in Australasia, parts of Europe and with limited installations in the USA, does not directly measure, or estimate the size of a queue. The lack of knowing the queue size often exceeds the offset algorithm's ability to discharge the queue before the upstream platoon arrives, thus creating shockwaves and magnifying the congestion effect [2].

This paper specifically investigates whether the length of queues can be accurately estimated from single loop detectors positioned at the stop line. The primary objectives of this paper are two-fold:

1. Identify variables that are suitable to estimate queue lengths using empirical data from an urban arterial road;

2. Propose a queue prediction model that can use data from a stop line positioned inductive loop detector;

The paper is organised as follows; the first part described the background and introduces the objectives of this paper; the second reviews the literature and analytical methods; the third part describes the data and details the methodology applied to investigate the problem; the fourth part outlines the results of the methods applied to develop a conceptual queue estimation model; the fifth part discusses the further work required to generalise the concept, and the last part summarises the conclusions of the research.

\section{LITERATURE REVIEW}

\subsection{Queue Estimation}

Over the past few decades, various types of queue models have been developed such as the cumulative input- 
output forms [3], limited by long queues [4] and the detector position; travel time using mobile sensors to reconstruct the queues [5]; and traffic shockwaves based on continuum flow theory relying on comprehensive input information which often cannot be collected at the given time. Other include linear regression models requiring constant traffic cyclic flow conditions, or aggregates detector data, but this smooths variations too much and reduces accuracy of detecting the back of the queue during highly fluctuating flow conditions [4].

The definition of a queue varies considerably as well, it ranges from vehicles that were stationary at the start of the green, some also include vehicles that joined the back of queue after the green started, other describe all vehicles travelling in a moving queue below $5 \mathrm{mph}$ as a queued vehicle [6]. Liu et al. [4] stated that vehicles in a moving queue are those that join the queue after the rear of the original (standing) queue starts to move. For the purpose of this paper, a queued vehicle is one that was queued during the red phase and those that joined during the green phase that were impeded by the back of the discharging queue are classified as queued.

Given the accuracy of the data that can be extracted from a single loop detector, Liu et al. [4] proposed a prediction model, but it relies on a detector loop upstream of the stop line, applying shockwave theory to determine the different flow states. Furthermore, their methodology relies on highresolution data, and event-based data that collect phase changes and detector actuation. Typically, SCATS also provides this type of data in high resolution. High-resolution data is used to identify "break points" in the traffic flow that may indicate the difference between the queued vehicles and those joining the back of the cleared queue, and platoonbased upstream vehicles. A similar process using stop line detectors to identify breakpoints between queued and platooned vehicles was investigated.

\subsection{SCATS}

The SCATS algorithm is described in detail in Sims and Dobinson [1] and Lowrie [7]. A critical assumption in SCATS is that it uses the time gap between vehicles and applies a steady state traffic flow relationship [8] to estimate the speed at the stop line. This estimated speed value is applied as a proxy for determining saturation conditions. Neither headway nor occupancy time is a suitable parameter as it can vary between more than one speed value [1].

Offset plan values in SCATS are mostly based on observed average travel time, or an assumed constant travel speed between intersections. The general use of average cruise speeds $[9,10]$ does not accurately capture the exogenous influences and variation in driver behaviour in response to the road, geometry, or prevailing traffic environment [11]. These influences are exhibited via the individual and platoon cruise speed variations, influenced by queue discharge, mid-block lane changing, route selection, driver anticipation of signal phasing, and other motivation or distraction factors [12-16].

\subsection{Speed from Single Loops}

Estimating speed from single inductive loops is prone to error in mixed flow regimes due to the length of vehicles on single inductive loops influences the estimate $[1,17]$ and is not very useful in real-time traffic management applications $[17,18]$. Nonetheless, for SCATS loops Sims and Dobinson [1] stated that where flows are continuous over the entire cycle, the average vehicle length could be derived from the occupancy data and hence the average speed re-estimated within the SCATS algorithm. The true error can only be estimated using field data collected from the SCATS system.

\subsection{Headways}

Previous research focused on the mean headway, found that the queue length causes shorter saturation headways [19]. Jin et al. [20] found the corresponding mean values based on queue position level out gradually, typically after the fourth or fifth vehicle. However, Li and Prevedouros [21] found headways still decreased up to around the 12th queued vehicle. $\mathrm{Li}$ and Prevedouros [21] studied the differences between long $(>20)$, medium $(12 \leq$ queue $\leq 20$ vehicles $)$ and short queues $(<12)$ and found that the last few vehicles in a long queue may produce elongated or compressed headways. Examining the variation of headways could form a crucial part to differentiate between queued and platooned vehicles. More recently based on the data collected from several intersections in Auckland, Chaudhry et al. [22] reported that headways at stopline continue to decrease even beyond $12^{\text {th }}$ vehicle in the queue. Chaudhry and Ranjitkar [23], Chaudhry and Ranjitkar [23, 24] proposed new methods to estimate capacity and delay at signalized intersection for cases with decreasing headways.

\section{METHODOLOGY}

The objective of this research is to determine if continuous input data measured from an inductive loop at the stop line can be used to accurately predict the queue membership status of the vehicle when it passes over the loop. In order to develop a model for predicting queue state, which is a categorical variable, binary logistic regression was selected to determine the probability of a vehicle being queued or platooned. Logistic regression is a part of the category of generalized linear models. The generalised model form for a logistic regression equation is given as:

$P(Y)=1 / 1+e^{-\left(b_{0}+b_{1} X_{1}+b_{2} X_{2}+\cdots b_{n} X_{n}+\varepsilon_{i}\right)}$

where,

$P(Y)$ : Probability of $\mathrm{Y}$ occurring

$b_{n}$ : Regression coefficient

$X_{n}$ : Predictor variables

Logistic regression makes no assumption about the distribution of the independent variables. They do not have to be normally distributed, linearly related, or of equal variance within each group. Similar applications of binary logistic regression to describe traffic flow states can be found in $[25,26]$. 


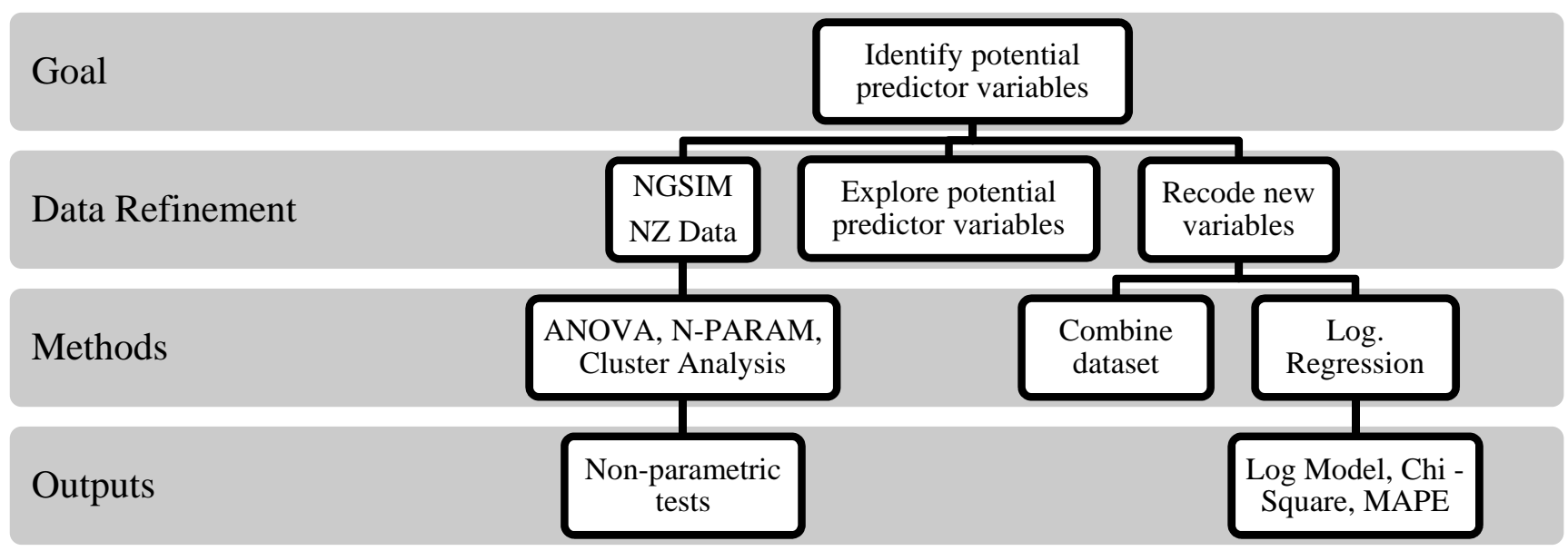

Fig. (1). Research structure.

Fig. (1) shows the outline of the methodology applied to develop a predictive model. The goal was to develop a predictive queue model by first exploring the data to determine which variables are suitable as predictors that best explain the variability in queue flow dynamics. The data were checked for normality after which appropriate inferential tests were applied to determine significant differences between groups of data to identify if cut points between queued and platooned vehicles were discernible. The data refinement process provided new variables that may be better at explaining the observed variation in the data.

\subsection{NGSIM Data}

The Federal Highway Administration's Next Generation Simulation (NGSIM) program has made available a detailed vehicle trajectory dataset for researchers. Data used in this paper were collected from Peachtree Street, an arterial primarily running north-south in Atlanta, Georgia. The speed limit on the Peachtree Street is $35 \mathrm{mph}$. A project by the University of Idaho [27] developed new insights from an analysis of the original NGSIM dataset [28].

Some corrections were made to the dataset such as removing negative speed, and correcting the stop line (bar) locations. The Statistical Package for Social Scientists (SPSS) format dataset represents vehicle trajectories on a segment of Peachtree Street in Atlanta, Georgia collected between 12:45 p.m. and 1:00 p.m. (set A1) and between 4:00 p.m. and 4:15 p.m. (set A2) on November 8, 2006. There are five intersections; four are signalised, and one is unsignalised.

\subsection{Data Refinement}

The first task was to identify suitable subsets of trajectory data that had at least three vehicles either queued or platooned at the stop bar so that the flow parameters can be extracted and analysed between queued and non-queued vehicles as they passed over the stopbar. The filtered dataset provided the necessary visual graphing (Fig. 2) of the trajectory data showing its longitudinal progression at 0.1 -second time steps. It shows the vehicle schema used to identify those vehicles queued and those that join as a platoon after the last queued vehicle, together with the signal state.

To emulate the collection of vehicle data from a SCATS detector loop, the data were filtered to represent 20 feet ( 6 $\mathrm{m}$ ) of trajectory records immediately upstream (Fig. 2) of the stopbar that corresponds to a typical SCATS detector loop location at the stop bar and the most frequently occurring vehicle length of 15-20 feet [6]. Trajectories from 123 vehicles, queued and non-queued were extracted for analysis in SPSS over 17 signal cycles spanning both the interpeak and afternoon peak periods from the northbound dataset only.

The subset of data was extracted from the NGSIM database from lane 1 and 2, as these represent straight ahead movements, which often had the longer queues present. Fig. (3) shows the dimensions of the variables.

Data were selected from only the green phases that contained more than three vehicles in a queue or platoon per cycle. Additional variables were coded such as;

Space Gap (ft) = Spacing between following and preceding the vehicle(ft.) - Preceding vehicle length (2) (ft.)

Spacing is the space headway between the front of a vehicle and the front of a preceding vehicle; the space gap thus represents the physical distance between the two vehicles.

Follow-up Headway (s) $=$ (Vehicle FrameID Preceding Vehicle FrameID) * 0.1

In keeping with the need to use parameters that can be directly measured from inductive loops, the occupancy for an equivalent standard SCATS detector loop of length $14.7 \mathrm{ft}(4.5 \mathrm{~m})$ [29] was calculated from the trajectory dataset using the measured speed (Vehicle Speed) of the vehicle and adding the vehicle length to provide an effective vehicle length passing over a virtual loop at a fixed point, at a given speed. 


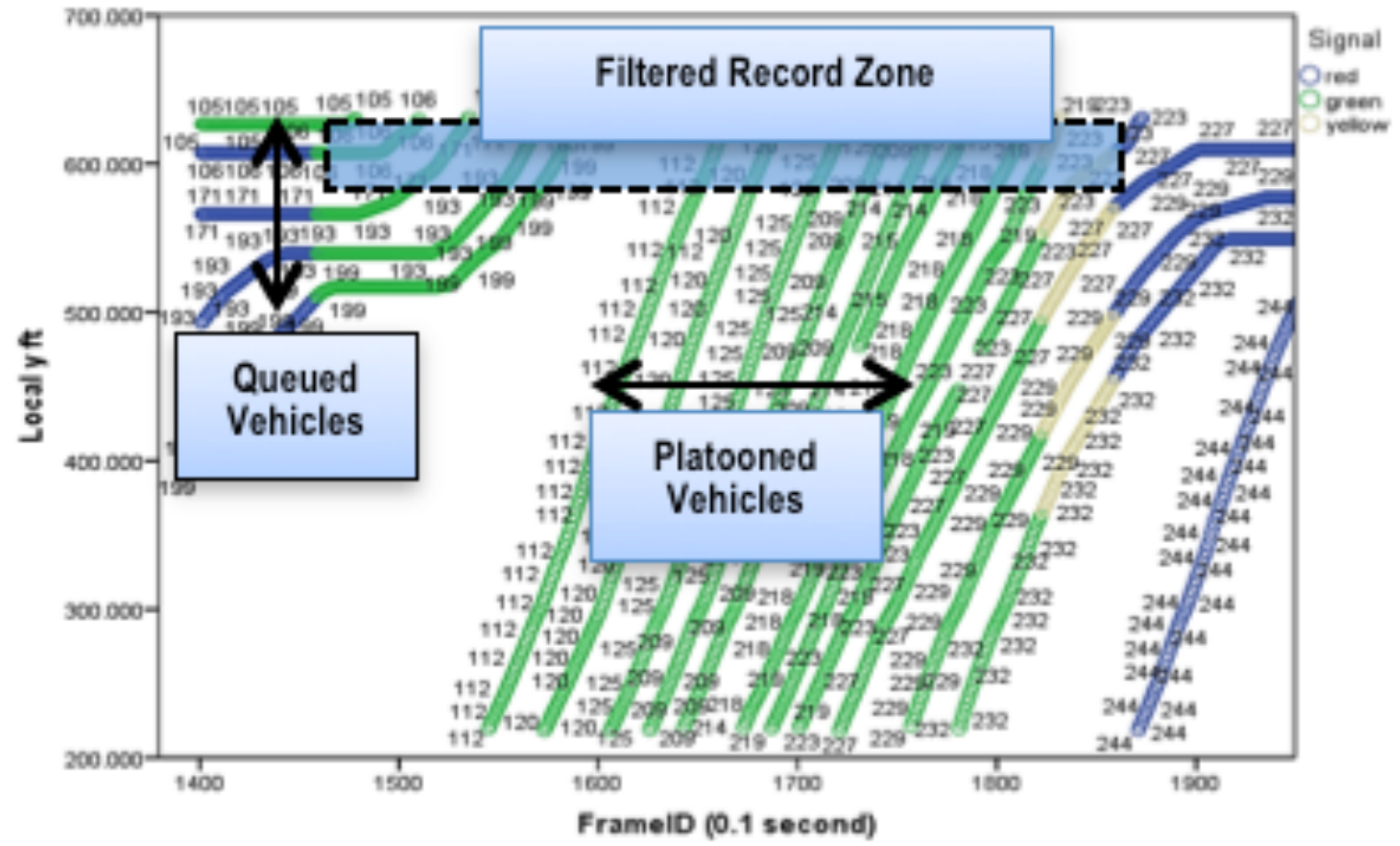

Fig. (2). Area of subset of records extracted.

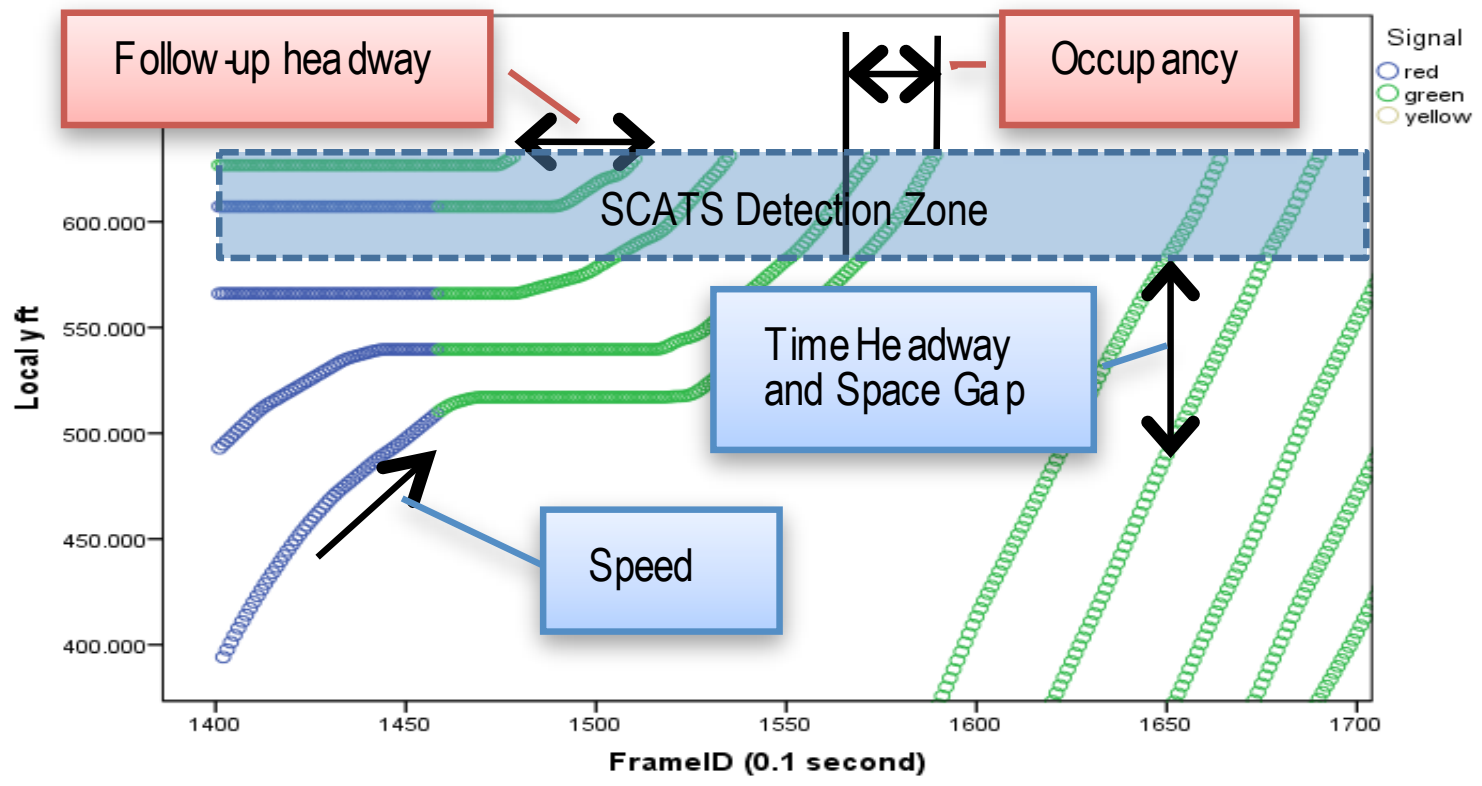

Fig. (3). Dimensions of variables.

$$
\text { Occupancy }(\mathbf{s})=\frac{(14.763+\text { vehicle length })}{\text { vehicle speed }}
$$

The first step of the analysis was to extract the following variables for screening and normality tests and to apply an ANOVA test on the selected variables, such as; Vehicle Speed (mph), Spacing (ft.), Time Headway (s), Space Gap (ft.), Follow-up Headway (s), Occupancy (s).

The first analysis set out to determine the properties of the trajectory variables between queued and platooned states (Fig. 4). The second analysis was centred on determining if the breakpoint between the last vehicle in a queue and the following platoon leader is a significantly different value that can be easily detected and discriminated. The dataset was analysed to establish the properties of the variables that can be directly collected, or derived, from a single loop detector, such as:

1. The variability between queued and non-queued vehicle speed, space headway, time headway, followup headway, space gap and occupancy values,

2. The variability of the above variables between the last queued vehicle and the following platoon leader, and

3. The variability of the parameters disaggregated by queue position. 


\subsection{Properties of Data at the Stop Line}

The NGSIM dataset used video extraction of the vehicles by recording their properties at $0.1 \mathrm{sec}$ intervals. There are some known errors in the dataset [30], and these were eliminated and filtered out in the extracted records. The data were screened to check for normality assumptions and outliers and were inspected to examine the variability by vehicle position from the start of the green phase (Fig. 4a, b). Vehicle Speed showed the clearest distinction between queued and platooned vehicles. The Vehicle Speed parameter appears to be a good predictor in determining the queued status of a vehicle crossing the stop line detector.

\subsection{Model Development}

Previous researchers attempted to use the variability of observed parameters between successive vehicles to determine distinctive breakpoints in the traffic flow that would differentiate the back of the queue from joining platoons of vehicles. This study investigated the variation of the flow parameters relative to the queue position to determine whether the variation between those queued, and those that are platooned, indicates queue membership status. The data used for this study had queue lengths of between 2 and 11 vehicles, and thus should only be generalised within those limits until independently verified with data of longer queue lengths.

\section{RESULTS}

The data are mostly non-normally distributed requiring the use of non-parametric analysis techniques. The nonparametric equivalent test for 2-independent samples is the Mann-Whitney test [31]. The results of the non-parametric tests analysing the variance between the means of queued and non-queued vehicles are summarised in Table 1. The variables of Vehicle Speed, Space Headway, and Space Gap show significant differences in the mean scores between queued and non-queued platoons. The data were recoded to reflect if the vehicle was either queued at the start of the

(a)

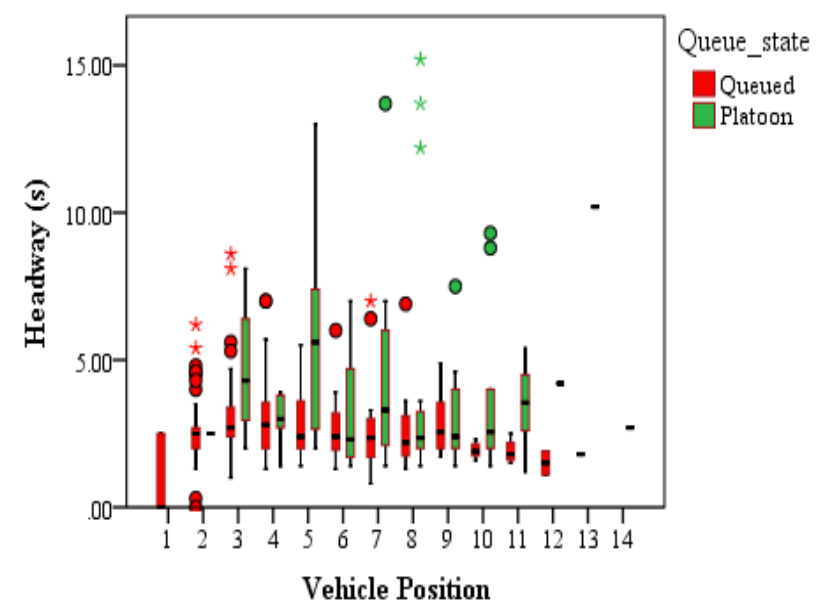

green and joined the queue during the green, or part of the platoon joining the back of the queue once the last queued vehicle discharged over the detector zone. The traffic state can be expressed as a function of the following variables:

Traffic state $=f$ [speed, headway, spacing, vehicle position, signal state]

A binary logistic regression was run applying various methods of variable entry. As there is no preceding research to select the order or importance of likely predictors, a small sample $(n=123)$ from only the northbound traffic was used as a block entry of all the variables as main effects.

Using the Wald statistic as an indicator of which variables are stronger predictors, various models using the backward stepwise method were selected with the stronger predictors of Vehicle Speed and Follow-up Headway as the first entry block, and Occupancy, Space Gap and Time Headway as the second entry block. Based on these results, a larger sample $(n=346)$ from both the north and southbound traffic was extracted to determine the best model predictors, including using a vehicle position as both a categorical variable, as well as an interaction term with speed and headway. The larger sample improved the predictive accuracy, but the goodness of fit suffered from the increased sample, as the Hosmer \& Lemeshow test can be conservative with continuous variables. A number of models were re-run using a random selection of $50 \%$ of the data extracted $(n=172)$, and this repeatedly showed improved goodness-offit statistics, as well as improved the accuracy over the previous models. Generally occupancy did not contribute much to the predictive power of the model, whereas including vehicle position improved both accuracy and model fit. The results of the best four model fit tests are summarised in Table 2 . The summary shows that the best model (3) correctly classifies a vehicle as queued or platooned $94.8 \%$ of the time. It also has the highest $\mathrm{R}^{2}$ values of all models that were analysed. However, it is more complex as it includes Vehicle Position that was recoded into 3 bins, short (1-5), medium (6-9) and large (>9). The accuracy is measured as the absolute difference error between the number of observed and predicted queued

(b)

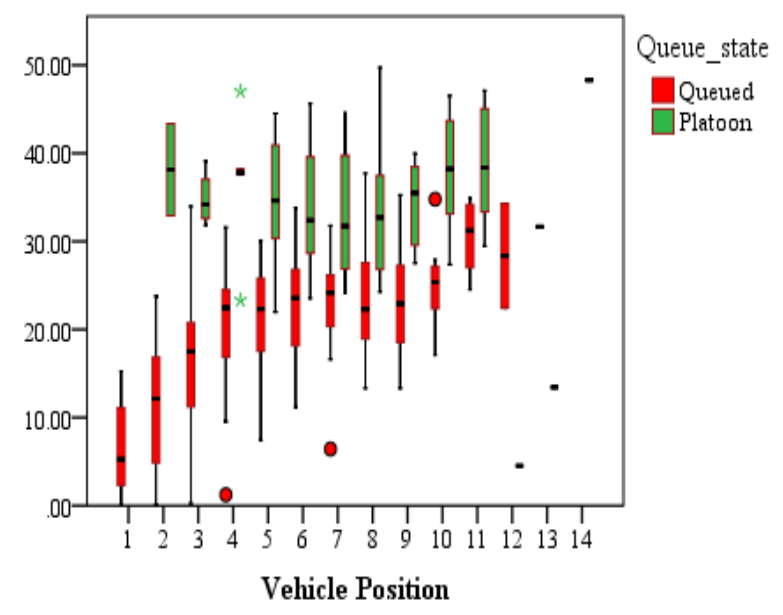

Fig. (4). Variation by vehicle position (a) follow-up headway (b) of vehicle speed. 
vehicles at each cycle, expressed as a percentage of all the observed queued vehicles. (Equation 6)

Accuracy (\%) $=\frac{\sum A B S\left(Q_{o b s}-Q_{\text {pred }}\right)}{Q_{o b s}} * 100$

where,

$Q_{o b s}:$ Observed number of queued vehicles

$Q_{\text {pred: }}$ : Predicted number of queued vehicles

It is worth mentioning that of the four variables, Vehicle Speed has an undefined error component due to its reliance on using an estimated average vehicle length to derive speed. However, SCATS goes through a self-calibration process when the highest flow in a period is recorded, thus the effective vehicle length is updated at critical intervals. For testing sensitivity a new variable Error Speed was calculated by applying a random error term to the Vehicle Speed variable Arendonk (1996) cited in [32] a test using the average error of $12 \%$ for an unbiased estimator of speed was randomly applied to the observed vehicle speed.
Model 1 was re-run substituting Vehicle Speed with Error Speed to determine the sensitivity of an error in inferring speed from a single loop detector, and revealed no significant difference in the predictive power. Given the objective of this research is to develop a queue prediction model using readily available data from a single loop detector, model 4 has the most appeal due to its simplicity of only relying on two significant variables, and this model's parameters Eq. (1) are reported in Table 3.

Fig. (5) shows the accuracy of model 4 applied to the observed queue length for each cycle from the NGSIM dataset. In order to improve the predictive accuracy of the equation an initial logical filter was applied to the data. Using the Excel SOLVER function and constraint values for speed the optimal accuracy results were obtained using Equation 6 if the speed threshold is between 0 and 28 fps. Equation 6 is only applied to the detector data measuring vehicle speed and follow-up headway for the values within the speed constraint. The predicted probability can be calculated for each vehicle, with values above 0.501

Table 1. Hypothesis test summary.

\begin{tabular}{|c|c|c|c|}
\hline & & Null Hypothesis & Decision \\
\hline \hline 1 & The distribution of Vehicle Speed ${ }^{1}$ is the same across categories of Q_State. & .000 & Reject the null hypothesis. \\
\hline 2 & The distribution of Space Headway ${ }^{2}$ is the same across categories of Q_State. & .001 & Reject the null hypothesis. \\
\hline 3 & The distribution of Time Headway is the same across categories of Q_State. & .094 & Retain the null hypothesis. \\
\hline 4 & The distribution of Space gap ${ }^{3}$ is the same across categories of Q_State. & .002 & Reject the null hypothesis. \\
\hline 5 & The distribution of Follow-up Headway is the same across categories of Q_State. & .521 & Retain the null hypothesis. \\
\hline 6 & The distribution of Occupancy $^{4}$ is the same across categories of Q_State. & .000 & Reject the null hypothesis. \\
\hline
\end{tabular}

${ }^{1}$ Queued vehicles have a significantly lower speed $(\mathrm{mph})(\mathrm{Mdn}=20.3)$ than platooned vehicles $(\mathrm{Mdn}=33)(\mathrm{U}=3219, \mathrm{z}=7.471, \mathrm{p}<.001)$.

${ }^{2}$ Queued vehicles have a significantly lower Space Headway $(\mathrm{ft}$.$) between vehicles (\mathrm{Mdn}=145.7)$ than for platooned vehicles $(\mathrm{Mdn}=204.8)(\mathrm{U}=2417, \mathrm{z}=3.285, \mathrm{p}=.001)$.

${ }^{3}$ Queued vehicles have a significantly lower Space Gap (ft.) between vehicles $(\mathrm{Mdn}=130.1)$ than platooned vehicles $(\mathrm{Mdn}=187.3)(\mathrm{U}=2344, \mathrm{z}=3.137, \mathrm{p}=.002)$.

${ }^{4}$ Queued vehicles have a significantly higher occupancy (s) than for $(\mathrm{Mdn}=0.770)$ than platooned vehicles $(\mathrm{Mdn}=0.527)(\mathrm{U}=860, \mathrm{z}=-4.849, \mathrm{p}<.001)$.

Table 2. Summary of model fit.

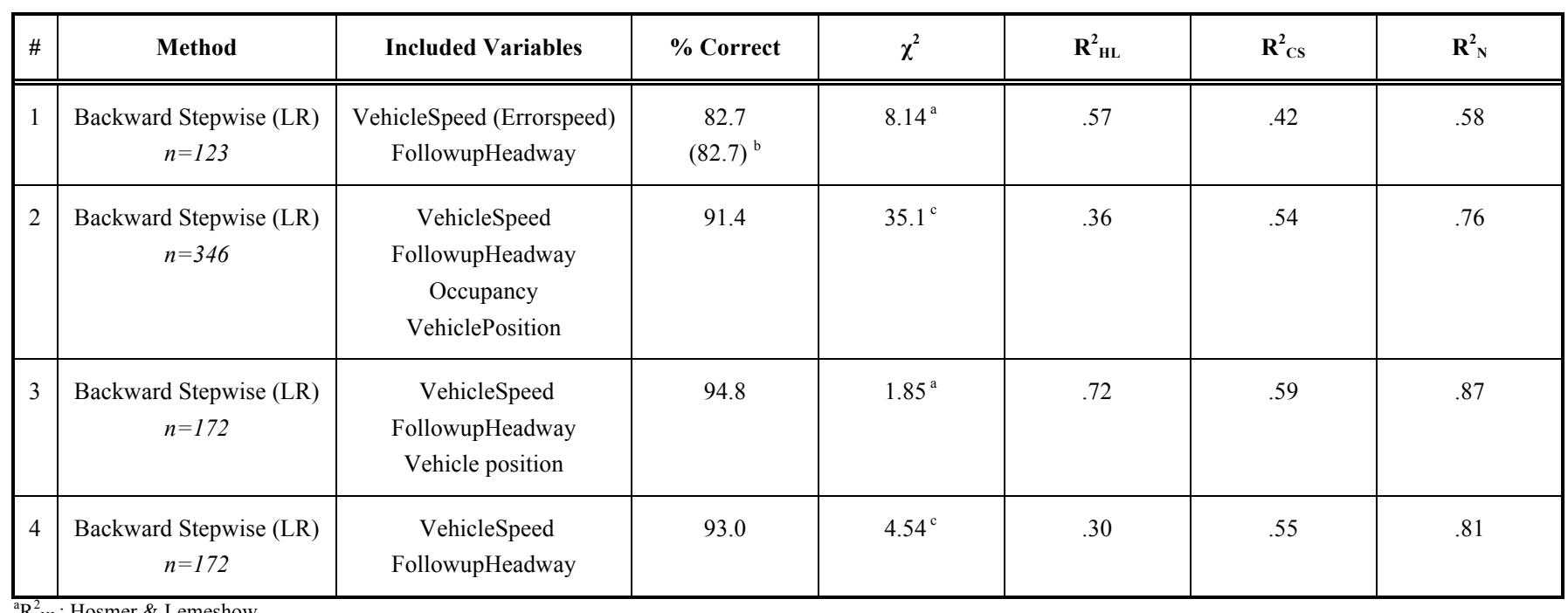

${ }^{\mathrm{a}} \mathrm{R}_{\mathrm{HL}}^{2}$ : Hosmer \& Lemeshow.

$* \mathrm{R}_{\mathrm{CS}}^{2}$ : Cox \& Snell.

$* \mathrm{R}_{\mathrm{N}}^{2}$ : Nagelkerke.

*The H-L model chi-square values are non-significant indicating a good fit of the data to the model.

${ }^{\mathrm{b}}$ Percentage Correct values in brackets are sensitivity checks using the estimated error in speed measured from single loops.

${ }^{\mathrm{c}}$ The H-L model chi-square values are significant indicating a poor fit. 
Table 3. Model 4 - Main effects variables in the equation.

\begin{tabular}{|c|c|c|c|c|c|c|c|}
\hline \multirow{2}{*}{ Variable } & \multirow{2}{*}{ B } & \multirow{2}{*}{ S.E. } & \multirow{2}{*}{ Wald } & \multirow{2}{*}{ Sig. } & \multirow{2}{*}{$\operatorname{Exp}(B)$} & \multicolumn{2}{|c|}{ 95\% C.I. for EXP (B) } \\
\hline & & & & & & Lwr & Upr \\
\hline Vehicle Velocity & .47 & .11 & 20.98 & .000 & 1.60 & 1.32 & 1.97 \\
\hline Followup Headway & .77 & .26 & 8.98 & .003 & 2.17 & 1.31 & 3.61 \\
\hline Occu-pancy & .37 & 1.63 & .05 & .817 & 1.46 & 0.06 & 35.12 \\
\hline Constant & -16.50 & 3.98 & 17.17 & .000 & .00 & & \\
\hline
\end{tabular}

classifying the traversing vehicle as platooned and values equal to and below 0.5 as queued.

A filtering algorithm can then be applied to classify a sequence of vehicles according to their predicted queue status. As it is possible that some misclassifications could occur, but by nature of a sequence of vehicles discharging over the stop line, the prediction can be improved by applying a logical filter as illustrated in Table 4. Typical logic values in a decision algorithm using combinations of vehicle position, measured speed, headway, and preceding and succeeding vehicle queue status can be then used to correct potential misclassifications of an individual vehicle's queue status.

The classification correction algorithm (Table 5) tests and corrects the predicted status of a vehicle by searching in a forward direction; first checking vehicle $n$ 's status and then

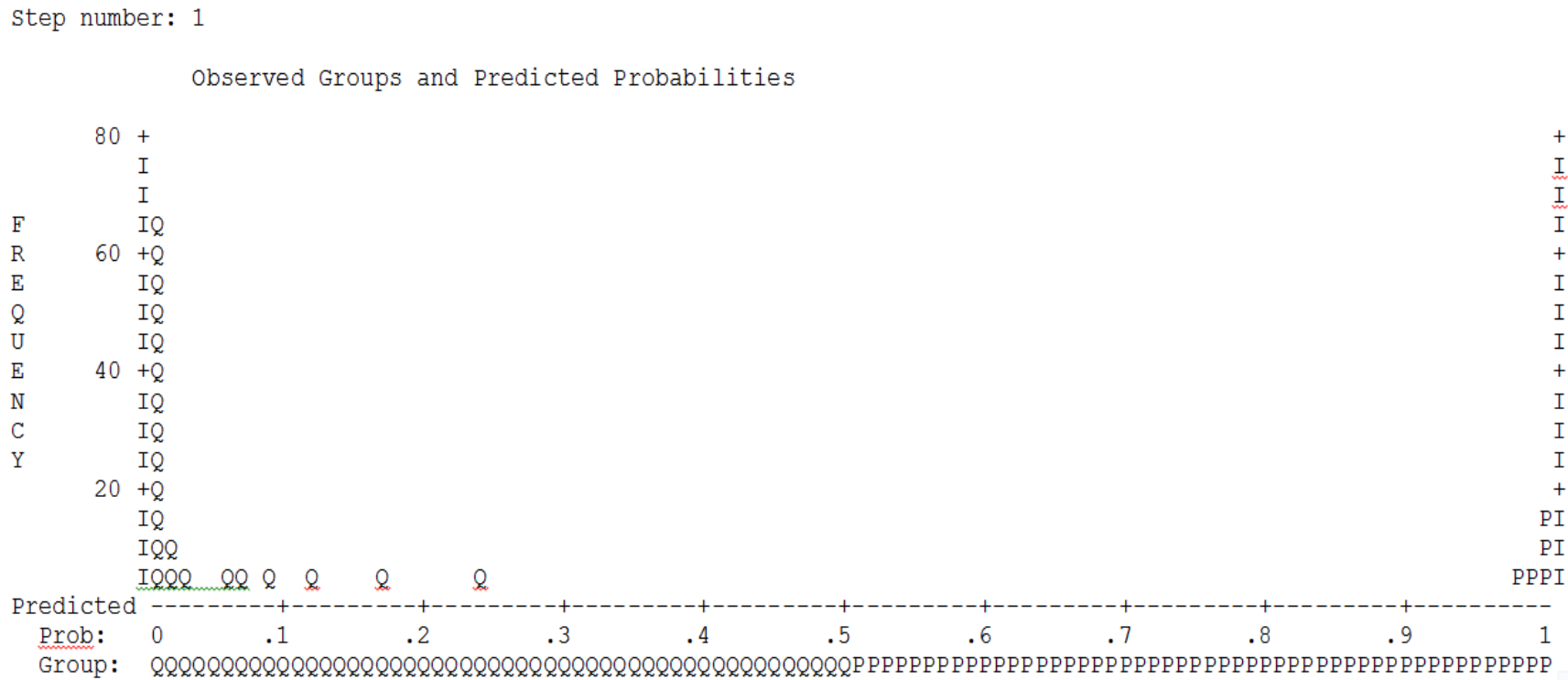

Predicted Probability is of Membership for Platoon

The Cut value is .50

Symbols: Q - Queued

P - Platoon

Each Symbol Represents 5 Cases.

Fig. (5). Observed Groups and Predicted Probabilities - Model 4.

Table 4. Hypothetical queue status correction results.

\begin{tabular}{|c|c|c|c|}
\hline \multirow{2}{*}{$\begin{array}{l}\text { Eq. } 6 \text { Queue Status Prediction for: } \\
\qquad V_{1}, V_{n+1}, V_{n+2}, V_{n}\end{array}$} & \multirow{2}{*}{ Correction } & \multicolumn{2}{|c|}{ Queue Length (in Vehicles) } \\
\hline & & Estimated & Corrected \\
\hline QQQPQQQPPPP & $\begin{array}{l}4^{\text {th }} \text { vehicle classified as Platooned, but unlikely due to following } \\
\text { vehicles 5,6,7 coded Queued - Correct } 4^{\text {th }} \text { vehicle }\end{array}$ & 6 & 7 \\
\hline QQQPPPQPPP & $\begin{array}{l}7^{\text {th }} \text { vehicle classified as Queued but unlikely due to preceding } 4,5,6 \\
\text { and following vehicles } 7,8,9 \text { coded Platooned - Correct } 7^{\text {th }} \text { vehicle }\end{array}$ & 4 & 3 \\
\hline
\end{tabular}


Table 5. Logical filter test.

\begin{tabular}{|c|c|c|c|c|c|}
\hline TEST & $\mathbf{n}-\mathbf{1}$ & $\mathbf{n}$ & $\mathbf{n}+\mathbf{1}$ & Adjusted $\mathbf{n}$ & Outcome of Logic Test \\
\hline \hline 1 & & & & & If speed $>28$ fps then $\mathrm{P}$, else \\
\hline 2 & $\mathrm{Q}$ & $\mathrm{Q}$ & $\mathrm{Q}$ & $=\mathrm{Q}$ & True value \\
\hline 3 & $\mathrm{Q}$ & $\mathrm{Q}$ & $\mathrm{P}$ & $=\mathrm{Q}$ & True - Possible cut point, check condition4 \\
\hline 4 & $\mathrm{Q}$ & $\mathrm{P}$ & $\mathrm{P}$ & $=\mathrm{P}$ & True - cut point confirmed \\
\hline 5 & $\mathrm{P}$ & $\mathrm{P}$ & $\mathrm{P}$ & $=\mathrm{P}$ & True - Platoon confirmed \\
\hline 6 & $\mathrm{Q}$ & $\mathrm{P}$ & $\mathrm{Q}$ & $=\mathrm{Q}$ & Change $\mathrm{P}$ to $\mathrm{Q}$ as the $\mathrm{P}$ is likely to be wrong \\
\hline 7 & $\mathrm{P}$ & $\mathrm{Q}$ & $\mathrm{Q}$ & $=\mathrm{Q}$ & Cannot occur as the $\mathrm{n}-1 \mathrm{P}$ would have already been changed in test 5 \\
\hline 8 & $\mathrm{P}$ & $\mathrm{P}$ & $\mathrm{Q}$ & $=\mathrm{P}$ & Keep as $\mathrm{P}$, the filter will correct the $\mathrm{n}+1 \mathrm{Q}$ to a $\mathrm{P}$ as in test 8 \\
\hline 9 & $\mathrm{P}$ & $\mathrm{Q}$ & $\mathrm{P}$ & $=\mathrm{P}$ & Change Q to $\mathrm{P}$, as $\mathrm{Q}$ is likely to be wrong \\
\hline
\end{tabular}

$n-1$ before $n+1$. This then corrects any misclassification of queues status of vehicle $n$ before moving to adjust vehicle $n+1$.

The NGSIM southbound trajectory data were used to check the accuracy of the model. The data included queues varying between 3 and 11 vehicles and a mixture of queued only, and queued with platooned vehicles. Each vehicle was manually classified as being queued or platooned. Those vehicles that were queued during the red phase and those that joined during the green phase that were impeded by the back of the discharging queue are classified as queued. The first and all subsequent arriving platooned vehicles that were not impeded by the back of the queue were all classified as platooned.

Each vehicle was arranged in their sequential departing order and their queue status calculated using model 4 . The logical filter test was then applied to the predicted queue status, and the final status was then compared to the observed field data. Table 6 shows a sample of one of the cycles used for validation to demonstrate the difference in accuracy of the prediction equation and that of the logical filter.

The validation dataset achieved a $97.5 \%$ accuracy of correctly classifying a vehicle as being queued. Validation field tests are underway using data from local intersections around Wellington, New Zealand, to further develop and validate the queue prediction model.

Fig. (6) shows the accuracy between observed and predicted queue length after the logical filter was applied for 27 southbound cycles. Cycle 13 was omitted as this cycle included queue failure under saturated conditions during the entire green phase.

\section{DISCUSSION}

This paper fits a broader study of understanding driver behaviour in response to queues at traffic signals. Understanding the influence and response to queues should lead to improving either optimisation of the traffic system, or could possibly be used to manage the system through the controlling of queue lengths to influence drivers' response to avoid queues. If we can estimate queue lengths from data collected from a single loop detector, traffic managers can create or dissipate queues to influence driver behaviour. The issue this paper addresses is the creation of a queue estimation model that uses data from a single loop detector.

Previous research shows headway stabilisation occurring between the $5^{\text {th }}$ and $12^{\text {th }}$ vehicle. The findings from the NGSIM data reveal that they do not stabilise after the $7^{\text {th }}$

Table 6. Sample extract of validation data set.

\begin{tabular}{|c|c|c|c|c|}
\hline Vehicle Position & Observed Q Status & Model 4 Predicted Q-Status & Logical Filter Correction & Q Accuracy \\
\hline \hline 1 & Q & Q & Q & Q \\
\hline 2 & Q & Q & Q & TRUE \\
\hline 3 & Q & Q Platoon & Platoon \\
\hline 4 & Q & Platoon & Platoon & \\
\hline 5 & Q & Q & Platoon & \\
\hline 6 & Q & Platoon & Platoon & \\
\hline 7 & Platoon & Platoon & Platoon & Platoon \\
\hline
\end{tabular}




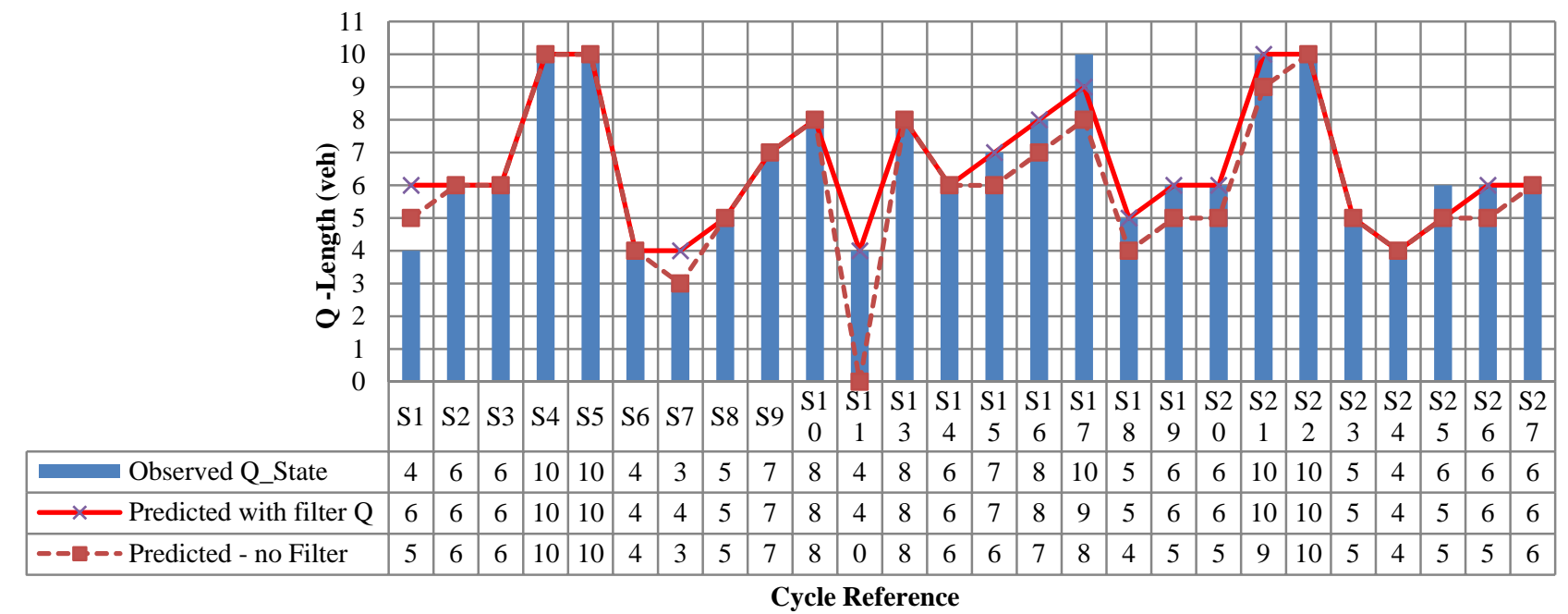

Fig. (6). Model 4 accuracy of SB Q-prediction.

vehicle, longer queues were not observed in this dataset to determine the point of stabilisation. Recent research on queue prediction used detectors placed some distance upstream of the stop line and used breakpoints in the occupancy as an indicator between the back of queue and arriving platoon [4]. Their models have limitations such as; the inability to determine the breakpoint when the arrival platoon headway is similar to the discharging queues headway. However, they argue this is a rare event. Other limitations are oversaturated conditions, and the reliance on an accurate, effective vehicle length, a typical problem with single loops. This paper does not deal with oversaturated conditions. The likely error in effective vehicle length, reveal that these error variations do not unduly influence the accuracy of the logistic regression model's prediction, and therefore are not thought to be a major influence in its application. However, the true effect of this error can only be tested using real data from a SCATS system, which is the subject of a further research study.

Once an individual vehicles queue status has been classified using model 4 for data where the speed threshold $<28 \mathrm{fps}$, the sequence of vehicles traversing a stop line the individual classification can then be corrected through a filtering algorithm to produce a most likely queue length estimate. This queue length can then be used for offset optimisation that considers the back of the queue position and its discharge time required to improve progression. Improved progression should result in reduced queue lengths and fewer cycle failures. Knowing the queue length can also be used to influence driver behaviour, such as increasing queues on selected movements to discourage those routes from being selected, and increasing the probability of a driver selecting an alternative route that has better progression and smaller queues on the approach to an intersection.

\section{CONCLUSION}

The Peachtree NGSIM high-resolution dataset was a good source of data to investigate the differences between the queued and platooned vehicles, however, the dataset lacks queues larger than 12 vehicles, and they discharged completely during each cycle. A total of 346 records from the IP and PM observations were extracted that had queues in excess of 3 vehicles, spread over 54 signal cycles. In addition to the 24 standard and 4 extended variables, a number of additional variables (Follow-up headway, space gap, phase time, vehicle position, and queue breakpoint) were coded to be used in further analysis and model construction.

The final set of six potential variables (Vehicle speed, Space Headway, Space Gap, Time Headway, Follow-up Headway, Vehicle Position and Occupancy) were inspected and all the variables except for speed data were distributed non-normal. Follow-up Headway for platooned vehicles was more variable than queued vehicles. Liu, et al. [4] argue that a similar headway between queued and platooned vehicles is a rare event. The analysis of variance of the NGSIM data shows that this often occurs and that the differences between queued and platooned vehicles are not significant.

The results of the non-parametric equivalent analysis of variance, Mann-Whitney tests, revealed significant differences between queued and platooned vehicles for Vehicle speed, Space Headway, Space Gap and Occupancy. A Logistic Regression model form was selected to develop a prediction of each vehicles likely queue/platooned state using the SPSS Binary Logistic Regression model with Vehicle Speed and Follow-up Headway as the strongest predictors. Of the $>20$ models analysed four different model forms and entry methods were reported in this paper, resulting in a predictive accuracy ranging from $83 \%$ to $95 \%$. The final logistic model form with only Vehicle Speed and Follow-up Headway was selected as the best predictive model at $97.5 \%$ accuracy on the fully extracted southbound dataset, as these two variables are easily measured by the SCATS detector loops. The strongest predictor of Vehicle Speed at the stop line is dependent on an estimated average vehicle length. A randomly applied error to vehicle speed did not markedly change the accuracy of the best prediction models.

The queue prediction model is based on queues that are $<12$ vehicles and therefore fall into the short queue range of 
prediction. It will require to be validated with longer discharging queues, however, it is not the intention to be validated for oversaturated conditions with queues blocking back. The models are further validated by an independent data set from the NGSIM database using data from the southbound direction. Further calibration and validation tests are planned to:

- Calibrate the offset model with a before-and-after experiment to measure improvement in progression and reduction in corridor delay using both a Saturn traffic model and field tests.

- Validate the queue estimation with more local collected data from four to six sites in Wellington, New Zealand.

\section{CONFLICT OF INTEREST}

The authors confirm that this article content has no conflicts of interest.

\section{ACKNOWLEDGEMENTS}

Declared none.

\section{REFERENCES}

\section{REFERENCES}

[1] A. G. Sims, and K. W. Dobinson, "The Sydney Coordinated Adaptive Traffic(SCAT) System: Philosophy and Benefit," IEEE Trans. Veh. Technol., vol. VT-29, no. 2, pp. 130-137, 1980.

[2] G. Abu-Lebdeh, and R. F. Benekohal, "Development of traffic control and queue management procedures for oversaturated arterials," Transport. Res. Rec.: J. Transport. Res. Board, vol. 1603, pp. 119-127, 1997.

[3] A. Sharma, D. M. Bullock, and J. A. Bonneson, "Input-output and hybrid techniques for real-time prediction of delay and maximum queue length at signalized intersections," Transport. Res. Rec.: $J$. Transport. Res. Board, vol. 2035, pp. 69-80, 2007.

[4] H. X. Liu, X. Wu, W. Ma, and H. Hu, "Real-time queue length estimation for congested signalized intersections," Transport. Res. C-Emer., vol. 17, no. 4, pp. 412-427, 2009.

[5] X. Ban, P. Hao, and Z. Sun, "Real time queue length estimation for signalized intersections using travel times from mobile sensors," Transport. Res. C-Emer., vol. 19, no. 6, pp. 1133-1156, 2011.

[6] M. Dixon, "Improved signalized intersection performance measurement," National Institute for Advanced Transportation Technology, University of Idaho, Moscow 2008.

[7] P. R. Lowrie, "The Sydney Coordinated Adaptive Traffic System Principles, Methodology, Algorithms," In: Int. Conf. Road Traffic Signal., London, 1982, pp. 67-70.

[8] J. G. Wardrop, "The Capacity of Roads," Oper. Res., vol. 5, pp. 1424, 1954.

[9] D. I. Robertson, and R. D. Bretherton, "Optimizing Networks of Traffic Signals in Real Time-The SCOOT Method," IEEE Trans. Veh. Technol., vol. 40, no. 1, pp. 11-15, 1991.

[10] M. Farzaneh, and H. Rakha, "Impact of Differences in DriverDesired Speed on Steady-State Traffic Stream Behavior," Transport. Res. Rec.: J Transport. Res. Board, vol. 1965, no. 1, pp. 142-151, 2006.

[11] M. Brackstone, and M. McDonald, "Driver behaviour and traffic modelling. Are we looking at the right issues?," In: Intel. Vehicles Sympos., 2003, pp. 517-521.
[12] R. Sipahi, S. I. Niculescu, and F. M. Atay, "Stability of traffic flow behaviour with distributed delays modelling the memory effects of the drivers," SIAM J. Appl. Math., vol. 68, no. 3, pp. 738-759, 2007.

[13] D. Stokols, R. W. Novaco, J. Stokols, and J. Campbell, "Traffic congestion, Type A behavior, and stress," J. Appl. Psychol., vol. 63, no. 4, pp. 467-480, 1978.

[14] C. J. D. Patten, A. Kircher, J. Östlund, L. Nilsson, and O. Svenson, "Driver experience and cognitive workload in different traffic environments," Accident Anal. Prev., vol. 38, no. 5, pp. 887-894, 2006.

[15] T. J. Triggs, and W. G. Harris, "Reaction time of drivers to road stimuli," Department of Psychology, Monash University, Melbourne 1982

[16] M. Brackstone, B. Waterson, and M. McDonald, "Determinants of following headway in congested traffic," Transport Res. F-Traf., vol. 12, no. 2, pp. 131-142, 2009.

[17] B. Coifman, "Estimating Median Velocity Instead of Mean Velocity at Single Loop Detectors," Transport. Res. C-Emer., vol. 11, pp. 211-222, 2003.

[18] B. Hellinga, "Improving Freeway Speed Estimates from SingleLoop Detectors," J. Transp. Eng-ASCE, vol. 128, pp. 58-67, 2002.

[19] K. Smilowitz, C. Daganzo, M. J. Cassidy, and R. L. Bertini, "Some observations of highway traffic in long queues," Transport. Res. Rec.: J. Transport. Res. Board, vol. 1678, pp. 225-233, 1999.

[20] X. Jin, Y. Zhang, F. Wang, L. Li, D. Yao, Y. Su, and Z. Wei, "Departure headways at signalized intersections: A log-normal distribution model approach," Transport Res. C-Emer., vol. 17, pp. 318-327, 2009.

[21] H. Li, and P. D. Prevedouros, "Detailed observations of saturation headways and start-up lost times," Transport. Res. Rec.: J. Transport. Res. Board, vol. 1802, pp. 44-53, 2002.

[22] M. S. Chaudhry, P. Ranjitkar, and D. J. Wilson, "Queue discharge behavior at signalized intersection: a comparison between field measurements with analytical and micro-simulation models," $J$. East. Asia Soc. Transport. Stud., vol. 9, pp. 1628-1643, 2011.

[23] M. S. Chaudhry, and P. Ranjitkar, "Delay Estimation at Signalised Intersections with Variable Queue Discharge Rate " J East. Asia Soc. Transport. Stud., vol. 10, pp. 1764-1775, 2013.

[24] M. S. Chaudhry, and P. Ranjitkar, "Traffic Signal Design with an Increasing Queue Discharge Rate," Asian Transport Stud., vol. 3 (In Press), 2015.

[25] W. Li, C. Li, X. Du, K. Qian, H. Zhang, and D. Hou, "A traffic flow prediction model based on ordered logistic regression," In: $6^{\text {th }}$ International Conference on Digital Content, Multimedia Technology and its Applications (IDC), Seoul, 2010.

[26] T. J. Gates, D. A. Noyce, L. Laracuente, and E. V. Nordheim, "Analysis of driver behavior in dilemma zones at signalized intersections," Transport. Res. Rec.: J. Transport. Res. Board, vol. 2030, pp. 29-39, 2007

[27] M. Dixon, A. Abdel-Rahim, and M. Kyte, "Improved simulation of stop bar driver behavior at signalized intersections," National Institute for Advanced Transportation Technology, University of Idaho, Moscow KLK712 N10-07, 2010.

[28] USDoT. (2008, October 31, 2011.). NGSIM - Next Generation Simulation. Available: http://www.ngsim.fhwa.dot.gov/

[29] AUSTROADS, "Guide to Traffic Engineering Practice: Part $7-$ Traffic Signals," R. Akçelik, Ed., ed. Sydney: Austroads, 2003.

[30] V. Punzo, M. T. Borzacchiello, and B. Ciuffo, "On the assessment of vehicle trajectory data accuracy and application to the Next Generation SIMulation (NGSIM) program data," Transport. Res. C-Emer., vol. 19, pp. 1243-1262, 2011.

[31] A. Field, Discovering Statistics using SPSS: SAGE, London, 2001.

[32] C. Sun, and S. G. Ritchie, "Individual vehicle speed estimation using single loop inductive waveforms," University of California: Irvine, 1999. 